\title{
REMOTE ELECTRONICS WORKBENCH - TAKING THE LAB HOME
}

\author{
Gustavo Ribeiro Alves ${ }^{1}$, António Cardoso ${ }^{2}$, José M. Martins Ferreira ${ }^{2}$ \\ ${ }^{1}$ Instituto Superior de Engenharia do Porto \\ R. Dr. António Bernardino de Almeida 431, 4200-072 Porto, Portugal \\ galves@dee.isep.ipp.pt \\ ${ }^{2}$ Faculdade de Engenharia da Universidade do Porto \\ R. Dr. Roberto Frias, 4200-465 Porto, Portugal \\ \{antonio.cardoso,jmf\}@fe.up.pt
}

\begin{abstract}
Students usually regard lab classes as the opportunity to practice learnt theory. Some would even pass more time in the lab performing new or the same experiment with slightly different parameters, if allowed to. Benefiting from the experience gained in the PEARL (Practical Experimentation by Accessible Remote Learning) project, the University of Porto has developed a Remote Electronics Workbench (REW) that allows students to carry out real experiments in electronics, from their home computer, through web-based access. The REW includes: interfaces to experimental scenarios; real video feedback from the lab environment; video-conference facilities to enable student-tostudent and student-to-tutor dialogue; and registration and booking pages to new and registered users, respectively. It is also a constituting part of a proposal to create a Remote Experimentation Network covering both European and Latin-American countries, under the Alfa II programme. Copyright (C) 2004 IFAC
\end{abstract}

Keywords: Educational aids, laboratory education, remote experimentation

\section{INTRODUCTION}

The concept of e-learning is now widely understood by the general educational community at all levels (i.e. at college and university levels), with a broad selection of solutions available both in the market and academia (Landon, 2003; Richards, 2001). Elearning has more recently benefited from the emergence of web-accessible labs providing support for a basic educational component: experimentation. The more recent concept of remote experimentation has been named in several ways (web-based remoteaccess laboratory, virtual or online laboratory, e-lab, etc.) throughout the last four to five years, in both conference and journal papers. For instance, Ko, et al., (2000; 2001a, b) use the expression "web-based virtual laboratory" in a series of articles that describe different experimental scenarios, developed at the National University of Singapore, that range from the control of relatively simple electronic circuits to more complex dynamic systems. Esche, et al., (2003) refers a series of "remote laboratories" (circa 30), while also referring that "the roots of such systems could be tracked back to a master-slave teleoperator developed at Argonne National Laboratory in 1954". The expressions "remote experimentation" and "remote lab" are used not only in (Esche, et al., 2003) but also in (Johnston, et al., 2001; Cooper, et al., 2002, Callaghan, et al., 2003; Mueller and Ferreira, 2003) and in several other references made by Esche, et al., (2003). The cited examples also provide a fair idea of the overall adoption of this supporting technology in distance education in different engineering fields (chemistry, civil, electronics, mechanical, mechatronics, etc.).

More recently, some authors have referred the pedagogical impact of remote experimentation and discussed ways of improving its benefits in a learning perspective. Terms such as "collaborative" and "cooperative", often associated with actions engaged by students in traditional lab classes, are being transferred into the remote experimentation arena, as seen in (Callaghan, et al., 2003; Ferreira, et al., 2002; Erbe and Bruns, 2003). 
The Faculty of Engineering of the University of Porto (FEUP) has been active in the area of remote experimentation since 1999, due to its involvement in several related European R\&D projects (Cooper, et al., 2002; Mueller and Ferreira, 2003). This paper describes the implementation of a remote electronics workbench (2004) that re-uses much of the lab infrastructure developed in the past PEARL project, while taking into consideration several functional and pedagogical aspects reported by students during the interim and final validation trials.

\section{BACKGROUND: THE PEARL PROJECT}

PEARL (2003) was a three-year R\&D project that ran from March 2000 till February 2003, with funds provided by the European Commission under the IST (Information Society Technologies) programme. In general terms, the PEARL project aimed to provide access to remote experimentation facilities in selected areas of science and engineering, namely biochemistry, fundamental physics, automatic visual inspection and digital electronics. Following a development phase that took place over the first two years, validation trials were carried out with students in Portugal, England, Scotland and Ireland. Paolo (2002) reported the feedback and comments received during the trials on aspects depicted in table 1 . The students' feelings were collected using both a questionnaire and an interview schedule. In total, Paolo analysed almost one hundred questionnaires and several hours of videotaped interviews.

\section{Table 1: Outline of the questionnaire for the user testing involving students}

Section Nature of questions

A: General Designed to gain an insight into the

Computing students' past experience of using
experience computers to determine if expertise has an impact on their use of the system or attitudes towards the system.

B: General This consists of 22 statements drawn questions from various usability instruments and about using covered how easy was it to learn how to the PEARL operate the system, usefulness of the system system and attitudes towards using the system. Each of the statements is responded to on a four-point Likert scale that measures how much the students agrees or disagrees with the statement.

C: $\quad$ This section asks students which of the

Collaboration collaboration tools they used and then tools

D: Learning with the PEARL system
Some of the main conclusions drawn from the analysis reported by Paolo in (2002) were:

- Real experimentation is not a replacement for simulation, but simulation alone is far less satisfactory than dealing with real devices (it is difficult to develop simulation models sufficiently sophisticated to cope with the unpredictability of real experiments and physical behaviour; students find real experiments more motivating than simulation, leading to better learning effectiveness)

- Synchronous communication facilities are of fundamental importance to enable the benefits of group work, particularly those related to good videoconferencing facilities - however, tutors should take into account that such resources will only be useful if enough bandwidth is available, which requires coordination with on-campus system administrators

- Scientific and pedagogical skills are more than ever required from tutors, who must be aware that script design must lead the students in such a way as to take full advantage of the powerful synergies resulting from distributed teamwork

Having in mind these conclusions, the FEUP team involved in PEARL decided to reuse the remote lab infrastructure developed under the project (after it had finished), for assisting in the lecturing of basic analog electronics concepts. The original idea was to provide students with an opportunity to repeat one or more experiments made on-site, i.e. during a traditional lab class, from their home computer. This idea addressed in particular the first conclusion pointed out by Paolo. The students do have the opportunity to simulate the circuit at home, using shareware such as the student versions of MultiSim or CircuitMaker. However, and as along as they have an Internet connection, students may now access the REW and control the very same circuit used during one of theirs lab class, repeating the experiment with the same or new circuit parameters (e.g. a different capacitor or resistor) and / or with other stimulus (e.g. a rectangular wave instead of a sine wave). Also, this initial motivation (enrich the pedagogical resources available to students) provided us with an excellent opportunity to update some aspects of the remote lab infrastructure.

The hardware part of the remote lab infrastructure, illustrated in figure 1, consists of: an experimental module (typically, a bread board with the circuit under experiment); a PXI system comprising the lab server (i.e. an embedded PC inserted in slot 0) and several test \& measurement instruments (function generator, oscilloscope, etc.); and also the web server (a standard desktop). The software part was updated to the latest version of LabVIEW, which enables swift generation of Virtual Instruments (VI) interfaces, remotely controllable through the web. This update allowed us to remove a third-party application from Nacimiento, named AppletVIEW, which was previously used for generating equivalent JAVA applets for the VIs, which enable direct access over the web via any common web browser, without 
requiring the installation of any plug-ins, as stated by Fidalgo in (2001). However, if the LabVIEW update enabled a serious breakdown in the development time (developing the JAVA applets required some time and effort), the counter part came in the form of the need to install a plug-in at the client side.

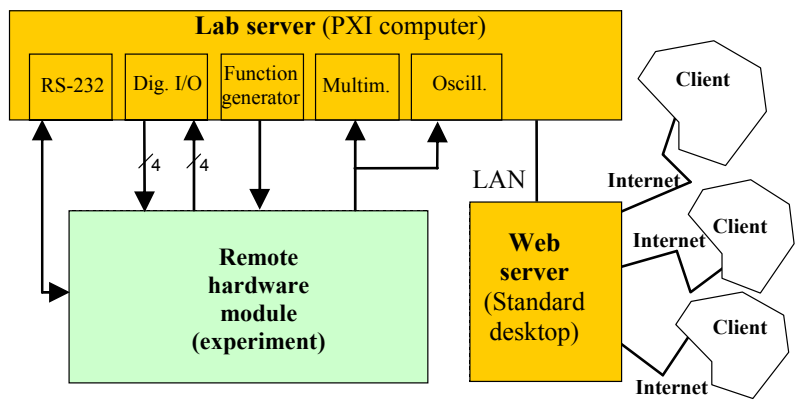

Fig. 1. The hardware component of FEUP's REW.

The $2^{\text {nd }}$ conclusion has also impacted the updating of the synchronous collaboration tools. In PEARL, we used CUSeeMe (2003) for audio / video conference, and although it served fairly as a collaboration tool, several reasons led to its replacement by FlashCom (2003). First, CUSeeMe uses UDP (Uniform Data Protocol), while FlashCom uses TCP/IP (Transport Connection Protocol / Internet Protocol). This difference is crucial in networks where UDP traffic is limited by network administrators, as it is also used for web-based radio broadcast. Second, CUSeeMe faced some problems with the firewall package installed at FEUP. This problem was solved after installing a new service pack, sent by the company responsible for the firewall. Additionally, the CUSeeMe server was installed in Dundee, Scotland. This meant that if two students placed in adjacent rooms, in Porto, Portugal, used CUSeeMe, all the corresponding UDP traffic had to be made via Dundee. Although this would go unnoticed in favourable conditions (enough bandwidth), some of the students reported difficulties in collaborating with each other, due to communication problems. To overcome all these problems, FEUP acquired and locally installed a FlashCom server.

Finally, the last conclusion led to a more careful design of the lab script. Considering that the students had already done the experiment in a traditional lab class, the on-line lab script could be simplified to a great extent, namely by reducing its total length to no more than two screens. This enables the students to cover the all script with just one or two mouse clicks on the side bar, instead of having to scroll up / down, which asks for more motivation, attention, and time to read it. Also the lab script is now presented in the entry page, alongside with the available options, instead of being just one link on the initial HTML page, as happened in PEARL. This caused some students to go directly to the experimental scenario, without even having seen the lab script.

Having clarified the background of the REW, we will now describe its current implementation, in more detail, in the following section.

\section{THE REMOTE ELECTRONICS WORKBENCH}

\subsection{The entry page}

Presently, the REW allows students to experiment a simple $2^{\text {nd }}$-order low-pass filter, namely by remotely controlling its input with a function generator and reading its output with an oscilloscope, both instruments being located at the lab. The access to the experimental scenario is made via an initial HTML page that displays the lab script, illustrated in fig. 2, and the tools selection menu that contains the following options:

- Access to Web cams placed at the lab (fig. 3)

- Access to video-conferencing

- Access to the remote lab:
○ Registration (required)
- Booking (required for control)
- Download plug-in (required)
$\circ$ Access to the current experiment

The on-line lab script guides the students through the remote experiment explaining: a) what is (are) the objective(s); b) what type of circuit and instruments are used; c) the rationale behind the experiment (e.g. if a certain input is applied or a set of conditions is satisfied, then a certain output or circuit behaviour should be expected); and d) what sort of conclusions or properties should be extracted from the circuit and / or experiment.

\section{FEUP's Remote Electronics Workbench}

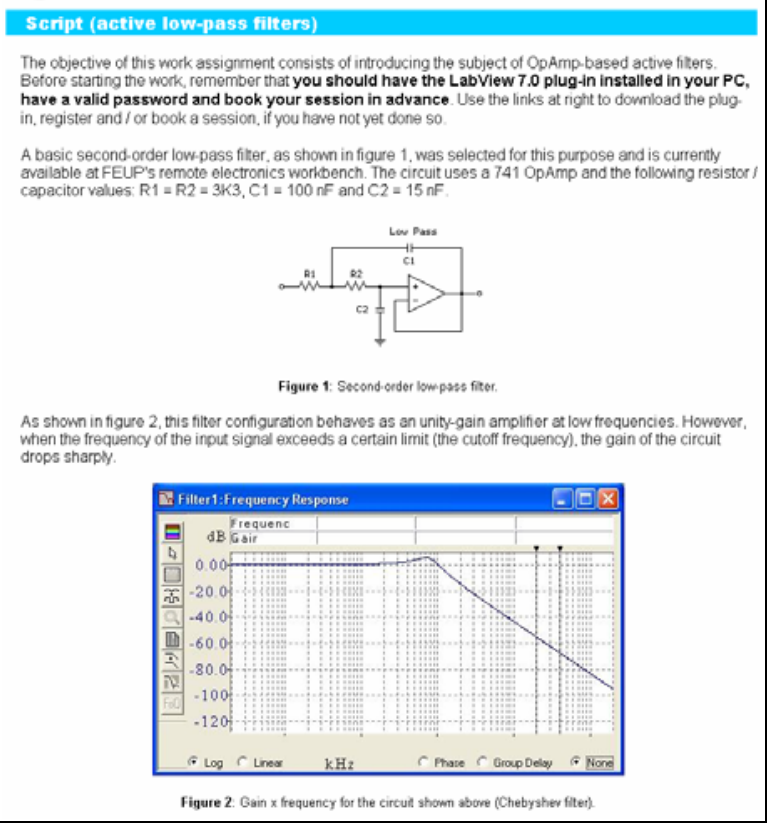

Fig. 2. The lab script contains explicit instructions on what is required and some schemes that help the students understanding the circuit under test.

\subsection{The video feedback from the lab}

The Web cams provide a live feedback from the lab, which is quite useful for a number of reasons: a) it provides a sense of reality, i.e. the students feel they are accessing something physical, as opposed to 
running a simulation; b) in certain circuits under test, it provides an image of the current status (LEDs on / off, position of switches, etc.). Both reasons were actually pointed out by students that participated in the first trials conducted under the PEARL project, as expressed by Paolo in (2002).

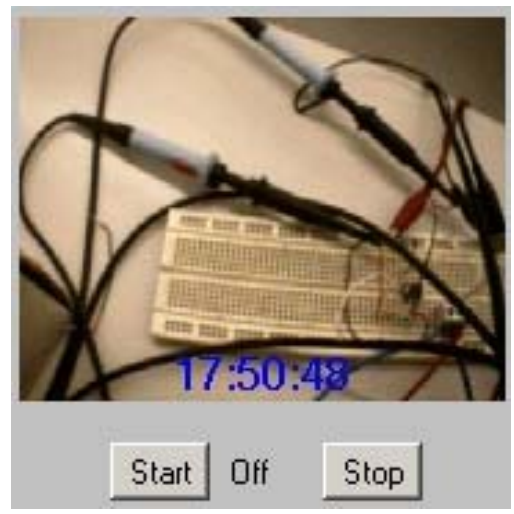

Fig. 3. Image of the circuit under test from one of the Web cams installed at the REW. The interface has start / stop buttons that allow reducing the bandwidth requirements for certain periods, while using the real-time clock as an indicator of the current status.

\subsection{Audio / Video communication}

As already stated, the audio / video communication is done through FlashCom, a synchronous collaboration tool that enables a student to establish contact with other students, the lab technician, or the tutor. The advantages of collaborative / cooperative work have already been extensively discussed in previous works, so that one may state this type of resource is vital in any remote experimentation environment. Interestingly, some students reported in PEARL a preferential use of the chat mechanism, as opposed to using the audio facilities, due to two reasons: 1) it is more reliable; and 2) when a tutor is present they may send private messages, so as to hide from the tutor any possible misunderstandings or mistakes that may cause a negative impact on him.

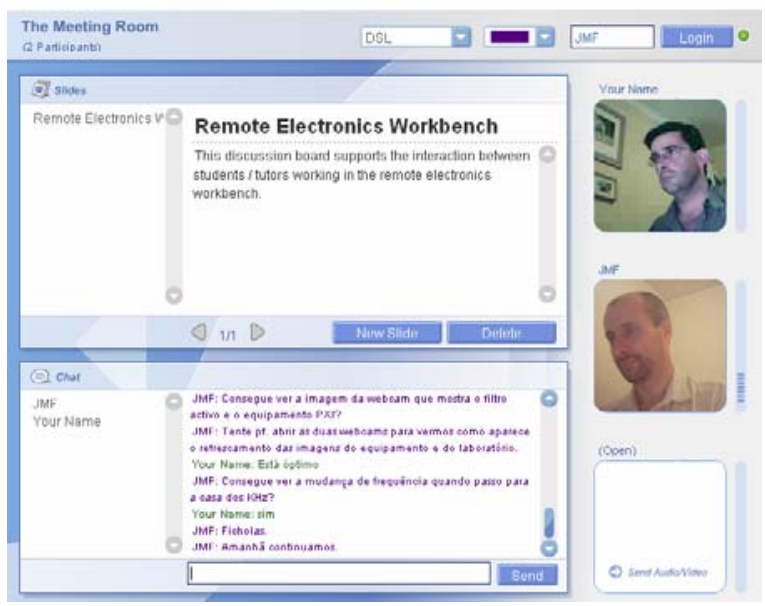

Fig. 4. Student and tutor using the video-conference tool, which contains two or more video frames, one slides window, and one chat window.

\subsection{Register / Booking mechanisms}

Before being able to access the remote lab (i.e. have a valid login / password), all clients are requested to register themselves with the lab administrator. This may be done through a simple HTML page (link "Registration" on entry page) that asks for some user identification data. After completing the form (some fields are mandatory), the client may either modify or submit it. Submitting the form causes the web server to automatically generate and send to the lab administrator an e-mail message, similar to the one illustrated in figure 5, who may then accept or refuse the registration request, using the two buttons visible at the message bottom. The decision will be reported to the client through an e-mail message, generated automatically by the web server.

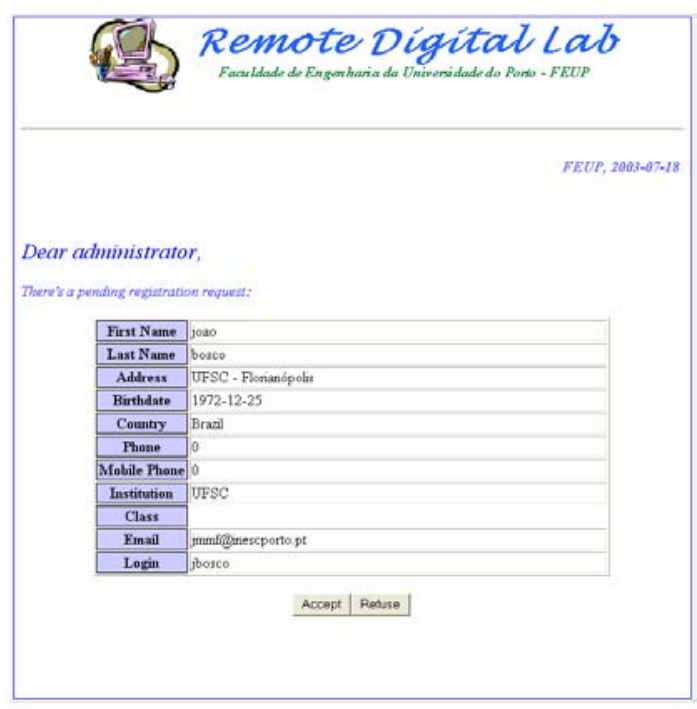

Fig. 5. A pending registration request, as received by the lab administrator.

Our solution for granting access and control to the remote experiment is based on two steps. First, it relies on a booking / scheduling mechanism that requires a valid login / password (obtained through registration, as previously described). The students are presented with a booking page that displays a 7day x 24-hours map, divided into 1-hour time slots, as illustrated in figure 6. A time slot may be booked by just clicking on it and entering a valid login / password on the small dialogue window prompted by the web server (fig. 6). Only the owner of a booked session or the lab administrator is allowed to free it again, afterwards, using the unbook option. A colour coding scheme indicates if a certain time slot is already booked, is under a booking request, is reserved for lab maintenance, or is free (a protection mechanism prevents booking elapsed time slots, according to the current web server time). Several students may access the lab during the same time slot, by sharing the same login / password (a sort of group key). Regarding control, and while conducting the experiment, only one student is allowed to control the remote instruments, at a given time, as imposed by the functionality of the associated VIs. However, the students are able to pass the control among them, as later explained in section 3.7. 


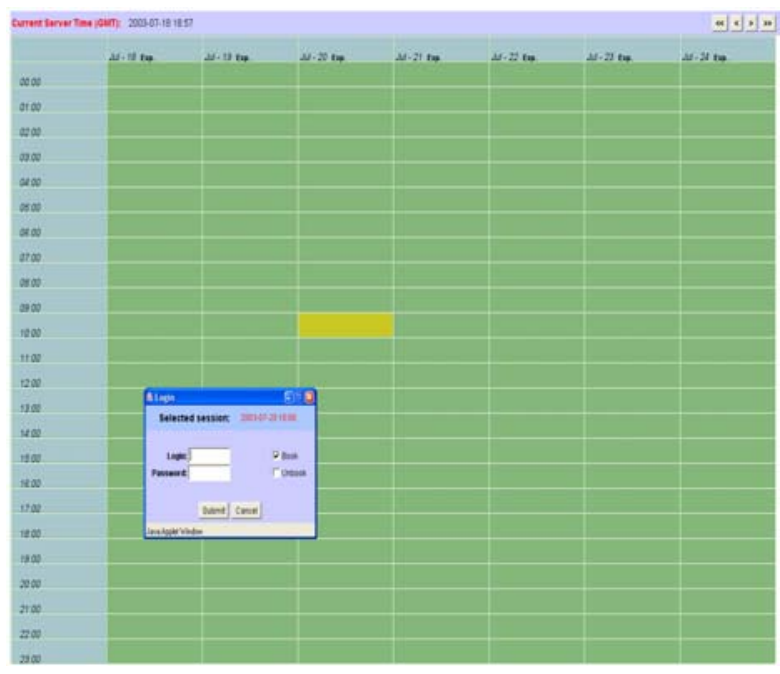

Fig. 6. The booking page while on the process of reserving a 1-hour time slot. The buttons on the top-right corner allow scrolling the visible map (7-day x 24-hours) day-by-day or week-by-week.

\subsection{Downloading / installing the plug-in}

Before accessing the remote lab, the students are required to download a plug-in (DataSocket Server), provided by National Instruments, in order to be able to control the test and measurement instruments, through the associated VIs. Once downloaded (about 20 Mbytes), the plug-in must be installed at the client computer, which necessarily requires administration privileges. These two details are at times frustrating to the students. For instance, when they are accessing the REW from their home computer for the first time, the size of the plug-in requires a significant time for downloading, even considering a fast connection (ISDN, or even ADSL). On other occasions, the students may not have administration privileges on the computer they are using, which generally happens on computers available at campus.

\subsection{Running the experiment}

Finally, having previously booked a time slot and having installed the plug-in, the students may then access the remote lab and run the experiment. Each access starts with the download of the VI panels, as illustrated in figure 7, which depicts the situation of using a computer connected to the Internet through a $56 \mathrm{~K}$ modem. Again, due to the size of the VI panels, this operation may take some minutes, and must be repeated every 1-hour time slot, i.e. even if two contiguous time slots are booked by the same student, the system will close the session on every hour turnover, thus requiring a new download, as illustrated in figure 8.

Downloading panel.

$4.15 \%$ of 788309 bytes.

Fig. 7. Downloading the VI panel(s) may take some minutes if the client is connected to the Internet through a low-speed link (e.g. a $56 \mathrm{~K}$ modem).
Fig. 8. Status bar indicating the current and ending times for a particular remote experiment session.

Apart from this system flaw, and once the VI panel is downloaded, the students gain control over the remote instrument by just clicking on it, as illustrated in figure 9. A request control command is sent to the server that immediately grants it. In working groups, if another student is already controlling the remote instruments, the system returns a warning message. In such occasions, the student may contact his / her group partner(s) - using the collaboration tools, request control, and obtain it as soon as the other student selects the "Release Control of VI" option. LabVIEW also incorporates a timing supervisor mechanism that releases the VI control whenever no actions are detected for a period of 5-minutes (default value). The timer is reset to zero if an action is detected within that period. The students may monitor this timer by selecting the "Show Control Time Remaining" option (shadowed in fig. 9).

The goal of the experiment currently available at the REW is to determine the cut-off frequency of a simple $2^{\text {nd }}$-order low-pass filter, based on an op-amp with a resistor / capacitor (RC) feedback loop. One process of determining the cut-off frequency is to apply several pure frequencies (sine waves), using the function generator, and check the circuit output with the oscilloscope (channel A connected to the function generator output $=$ filter input; channel B connected to the filter output). For frequencies below the cut-off frequency, the filter output follows the circuit input (circuit gain $=1$ ), while for frequencies above the output is attenuated (gain $<1$ ). This process requires the student to remotely control both the function generator and the oscilloscope, much in the same way as done locally in the lab class where the experiment is first introduced.

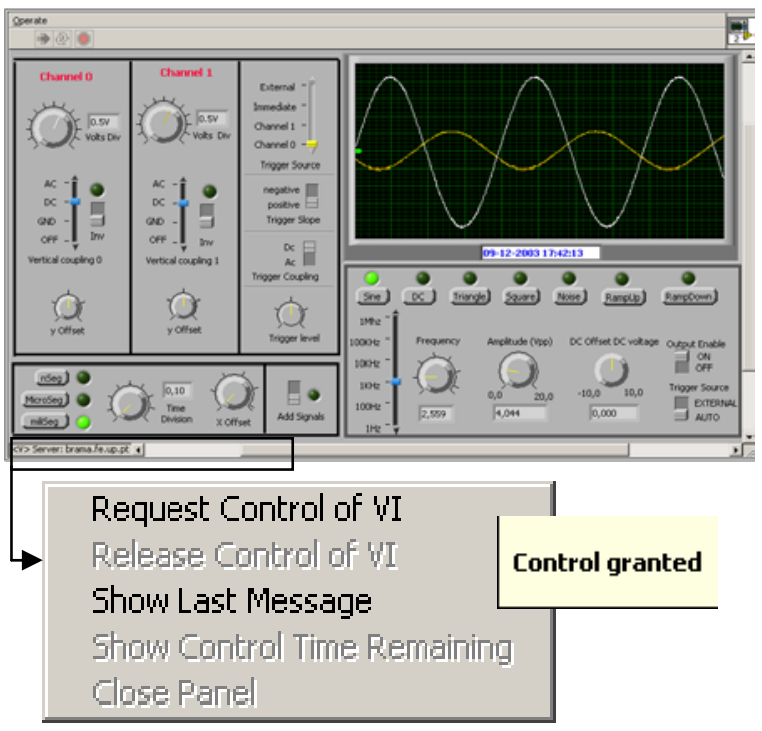

Fig. 9. Remote interfaces of the function generator (bottom-right frame) and of the oscilloscope (remaining frames). The bottom selection menu allows the students to request / release control of the corresponding VI. 


\section{CONCLUSION AND FUTURE DIRECTIONS}

The REW provides students with an opportunity to repeat an experiment made during a traditional class, from their home computer (as long as it is connected to the Internet), thus justifying the expression "taking the lab home". Although only one experiment is currently available (a simple $2^{\text {nd }}$-order low-pass filter, used in the lecturing of basic analogue concepts - or op-amp basic circuits), the remote lab infrastructure remains the same for any other experiments on electronics, provided they use the test and measurement instruments and resources already available at the lab server. Supporting a new experiment typically requires: a new circuit under test connected to the lab server; developing the VI panels for the experimental scenario (if more instruments are needed - apart from those already controllable through the existing VI panel, or if a different arrangement is preferable); designing the associated lab script; and updating the booking page with information on what experiment is available on each day.

Finally, the REW is also a constituting part of a proposal to create a Remote Experimentation Network (RexNet), covering both European and Latin-American countries, which includes a remote lab for experiments on mechatronics (Erbe and Bruns, 2003) and the RexLab (Alves, 2003), among others. The proposal was elaborated by a consortium comprising 10 institutions and submitted to the $8^{\text {th }}$ call of the Alfa II programme (Alves, 2004), which is expected to release the evaluation result during the $1^{\text {st }}$ quarter of 2004.

\section{REFERENCES}

Alves, R. Gustavo (2004). Remote Experimentation Network - Yielding an Inter-University peer-topeer e-service. Proposal to the Alfa programme. In: http://www.dee.isep.ipp.pt/ galves/alfa

Alves, João Bosco (2003). Remote Experimentation Laboratory (RexLab). In: http://rexlab.ufsc.br/.

Callaghan, MJ., J. Harkin, C. Peters, TM. McGinnity and LP. Maguire (2003). A Collaborative Environment for Remote Experimentation. In: Proceedings of the International Conference on Microelectronic Systems Education, Anaheim, California, USA.

Cooper, Martyn, Alexis Donnelly and José M. Ferreira (2002). Remote controlled experiments for teaching over the Internet: A comparison of approaches developed in the PEARL project. In: Proceedings of the $19^{\text {th }}$ annual conference of the Australian Society of Computers In Learning In Tertiary Education, Auckland, New Zealand.

CUSeeMe (2003). QuickNet. In: http://www.cuseeme.com

Erbe, Heinz-Hermann and F. Wilhelm Bruns (2003). Didactical Aspects of Mechatronics Education. In: Proceedings of the $5^{\text {th }}$ IFAC International Symposium on Intelligent Components and Instruments for Control Applications, Aveiro, Portugal.
Esche, Sven K., Constantin Chassapis, Jan W. Nazalewicz and Dennis J. Hromin (2002). A scalable System Architecture for Remote Experimentation. In: Proceedings of the $32^{\text {nd }}$ ASEE/IEEE Frontiers in Education Conference, Boston, MA, USA.

Ferreira, José M., Gustavo Alves, Ricardo Costa and Nick Hine (2002). Cooperative learning in a web-connected workbench. In: Lecture Notes on Computer Science 2438, "Groupware: Design, Implementation and Use", ISBN 3-540-44112-3, Springer, Proceedings of the $8^{\text {th }}$ International Workshop on Groupware, La Serena, Chile.

Fidalgo, André V., Ricardo J. Costa, José M. Ferreira and Gustavo R. Alves (2001). Experimenting the 1149.1 and 1149.4 test infrastructures in a Webaccessible remote Lab (without Plug-ins!). In: Proceedings of the $16^{\text {th }}$ Conference on Design of Circuits and Integrated Systems, Porto, Portugal.

Flash Communication Server (2003). Macromedia. In: http://www.macromedia.com/software/flashcom/

Johnston, Murray V., Frederick J. Cox, Gregory J. Forte, Dean C. Nairn, Richard S. Sacher, Anita Z. Schwartz, and Akos Vertes (2001). Remote Experimentation over the Net: Our First Year with MALDI. In: Analytical Chemistry, bimonthly Journal of the American Chemical Society, Volume 73, No. 15, pp 440 A-445 A.

Ko, C. C., B. M. Chen, J. Chen, Y. Zhuang and K. C. Tan (2001a). Development of a Web-based laboratory for control experiments on a coupled tank apparatus. In: IEEE Transactions on Education, Volume 44, No. 1, pp. 76-86.

Ko, C. C., B. M. Chen, S. H. Chen, V. Ramakrishnan, R. Chen, S. Y. Hu and Y. Zhuang (2000). A large scale web-based virtual oscilloscope laboratory experiment. In: IEE Engineering Science and Education Journal, Volume 9, No. 2, pp. 69-76.

Ko, C. C., B. M. Chen, S. Y. Hu, V. Ramakrishnan, C. D. Cheng, Y. Zhuang and J. Chen (2001b). A web-based virtual laboratory on a frequency modulation experiment. In: IEEE Transactions on Systems, Man, and Cybernetics, Part C: Applications and Reviews, Volume 31, No. 3, pp. 295-303.

Landon, B. (2003). On-Line Educational Delivery Applications: A Web Tool for Comparative Analysis. In: http://www.edutools.info/index.jsp

Mueller, Dieter and J. M. Martins Ferreira (2003). MARVEL: A mixed-reality learning environment for vocational training in mechatronics. In: Proceedings of the Technology Enhanced Learning International Conference, Milan, Italy.

Paolo, Terry Di (2002). Deliverable 9.2 - Plan of the Interim Validation, project PEARL IST-199912550. In: http://kmi.open.ac.uk/projects/pearl/

PEARL (2003). In: http://iet.open.ac.uk/pearl/

Remote Electronics Workbench (2004). Faculty of Engineering of the University of Porto. In: http://www.fe.up.pt/ jmf/rew

Richards, P. (2001). MIT to make nearly all course materials available free on the WWW. In: http://web.mit.edu/newsoffice/nr/2001/ocw.html 